\title{
Tribological Degradation of Head-Disk Interface in Hard Disk Drives under Accelerated Wear Condition
}

\author{
Yu Wang ${ }^{1,3}$, Xiongfei Wei ${ }^{2}$, Kwok-Leung Tsui ${ }^{3}$, and Tommy W.S. Chow ${ }^{1,4}$ \\ ${ }^{1}$ Center for Prognostics and System Health Management, City University of Hong Kong, Hong Kong \\ ${ }^{2}$ SAE Magnetics (H.K.) Ltd., Winnerway Industrial Area, DongGuan City, Guangdong Province, China \\ ${ }^{3}$ Department of Systems Engineering and Engineering Management, City University of Hong Kong, Hong Kong \\ ${ }^{4}$ Department of Electronic Engineering, City University of Hong Kong, 83 Tat Chee Avenue, Kowloon, Hong Kong
}

\begin{abstract}
To further increase the areal storage density of hard disk drive (HDD), one solution is to reduce the spacing between the magnetic head and disk to sub-1-nanometer regime. Such ultra-low spacing introduces great challenges to tribological reliability in the headdisk interface (HDI). Therefore, it is necessary to understand the characteristics and mechanisms of HDI degradation in the tribological condition. This paper investigates the degradation behaviors of HDI based on an accelerated wear experiment. Acoustic emission sensor and thermal asperity sensor were used simultaneously to monitor the touch down (TD) dynamics before and after each cycle of overdrive test in order to characterize the HDI degradation behaviors. Through analyzing the evolutions of HDI dynamic characteristics, the degradation mechanisms, including wear mechanism shift and HDI instability propagation, were figured out.
\end{abstract}

Index Terms - Head disk interface, tribological degradation, wear, touch down dynamics

\section{INTRODUCTION}

$\mathrm{T}$ HE NEED to store large quantities of data produced in the world requires intensive efforts to improve storage capacity of storage devices. As one of the most popular data storage devices, hard disk drives (HDDs) play a dominant role in modern data storage [1]. Further increasing the storage capacity of HDDs requires an ultrathin physical spacing between magnetic head (slider) and disk. Currently, head-disk spacing is controlled by a thermal fly-height control (TFC) technology [2], which employs a heating element (heater) integrated with the read/write transducer (Fig. 1). When a series of incremental powers are supplied to this heater, a small region around the read/write transducer will thermally expand towards the disk. With this technique in place, the slider flying-height can be adjusted flexibly. When the recording density is further increased to $1 \mathrm{~TB} / \mathrm{in}^{2}$, a clearance of sub 1 nanometer $(\mathrm{nm})$ between slider and disk is required [3]. At such low physical spacing, slider-disk contacts become inevitable, since HDD may encounter severe conditions such as vibration, shock, high temperature/humidity, and disk asperities during operation [4]-[7]. Slider-disk contacts lead to slider and disk wear that subsequently induces data corruption, slider/disk corrosion, and instable flying [8]-[10].

\section{FIG. 1 HERE}

The tribological characteristics of head-disk interface (HDI) have therefore received great interests in recent years. Extensive experiments of intermittent/continuous head-disk contact such as the flyability and durability test have been carried out to investigate the slider/disk durability [10]. The slider wear, after the test, was generally inspected by scanning electron microscopy (SEM), auger electron spectroscopy (AES) or atomic force microscopy (AFM) [2], [10], [11]. The fragile area of a slider is observed to be varied with the

Manuscript received X X, 2013 (date on which paper was submitted for review). Corresponding author: Y. Wang (e-mail: ywang95c(amy.cityu.edu.hk).

Digital Object Identifier inserted by IEEE designs of air bearing surface but mainly locates on the region of read/write element [12]. Disk wear appears to be less significant than slider wear due to its larger sliding area (whole circular track) [10] and lubricant protection [13]-[15]. The interaction between lubricant and slider is also raised as a hot topic since the slider dynamics induced by slider-lubricant interaction cannot be negligible under such a small recording regime. Optical surface analyzer (OSA) is a powerful tool for quantitative inspection of lubricant depletion and modulation [16]-[18], and thus has a number of successful applications in revealing the mechanisms of slider-lubricant interactions.

Despite the aforementioned tools for identifying HDI tribological damage, they only give the end results due to the destructive measurement. The end results may be incapable of providing sufficient information to identify the HDI degradation mechanisms that are crucial in guiding the enhancements of the slider/disk designs as well as in facilitating the extension of HDD life.

HDI degradation can be reflected by more than just mechanical damage (wear) to the interface; the vigorous slider vibration that can be used to characterize the instability of HDI system is also a significant sign of HDI degradation [19][21]. To gain a better understanding of the degradation mechanisms, this paper presented a technique to perform continuous monitoring for HDI degradation using AE sensor and thermal asperity (TA) sensor. To speed up the degradation process, an accelerated wear test by means of overdriving the TFC heater was carried out. Under such condition, the slider pole tip continues to protrude even after the touch down (TD) happens, making the slider pole tip contact with disk continuously. To characterize the HDI degradation, slider TD dynamics before and after each cycle of overdrive test were compared. Through analyzing the evolutions of slider dynamic characteristics, the degradation mechanisms including wear mechanism shift and HDI instability propagation were figured out. 


\section{EXPERIMENT}

\section{A. Experimental Setup}

The schematic of the experimental configuration is shown in Fig. 2. AE sensor and TA sensor were used to characterize HDI performance degradation via monitoring the touch down (TD) dynamics. AE sensor was also used to detect slider-disk contacts. TA sensor is an on-board sensor (Fig. 1), which has been proposed to map the disk defects [22], [23]. AFM and SEM were used to verify the final results. The test platform was the drive-based tester. The slider tested in this experiment was a typical pemto slider $\left(1.235 \times 0.7 \times 0.23 \mathrm{~mm}^{3}\right)$ covered by a $2 \mathrm{~nm}$ diamond-like carbon (DLC) film. The disk platter was a carbon coated 2.5-inch glass disk. The thickness of lubricant layer coated on the disk was $1.4 \mathrm{~nm}$. The disk rotation was driven by a spin stand with a rotating speed of $5400 \mathrm{rpm}$. A printed circuit board was used to control the heater power. The signals including TFC heater power, AE sensor, and TA sensor were simultaneously collected by a National Instrument acquisition system at each TD process.

\section{FIG. 2 HERE}

\section{B. Test Methods}

1) Test procedure

The testing procedure is as follows. 1) At the beginning, the slider was loaded onto track A of the rotating disk. A gradual increment of power is supplied to the TFC heater. AE RMS was calculated at each power step. When slider protrusion contacts with disk, TD power (TDP) can be detected once the AE RMS is greater than a predefined threshold $(130 \%$ of the initial AE RMS without touchdown). This TDP was used as a reference. The TD dynamics were also recorded. 2) The slider was then loaded onto a new track (track B). Then, the heater power was increased to TDP, denoted as $\mathrm{TDP}_{\mathrm{B}}$, and then set the heater power to $\mathrm{TDP}_{\mathrm{B}}+20 \mathrm{~mW}$ for one-minute overdrive test; 3) Slider was loaded back to the original track (track A) to redo the TD test. A new TDP and TD dynamics were recorded; and 4) Repeat 2) and 3) until 30 cycles were completed. It is worthy to note that the TDP in track B was updated every cycle.

\section{2) Characterization of Slider Wear}

In order to characterize the head wear, we introduced the TDP loss that is defined by the difference between the new TDP and the reference TDP in track A. Fig. 3 shows the TDP loss calculated by two TD processes: before and after an overdrive test.

\section{FIG. 3 HERE}

To verify the relationship between wear depth and TDP loss, a correlation study was carried out. Fifteen sliders were used in the overdrive test. AFM was used to measure the wear depth of the writer shield, which is easier to be worn than reader shield [3]. Fig. 4 (a) and (b) show the cross profiles of the writer shields in a fresh slider and a slider after overdrive test respectively. A distinct groove is observed in Fig. 4 (b), indicating an occurrence of serious wear.

\section{FIG. 4 HERE}

Fig. 5 shows the relationship between the TDP loss and the real wear depth measured by AFM. The R-square for the correlation is approximately 0.9 , indicating a perfect correlation. Therefore, we can conclude that the TDP loss can be used to quantify the slider wear.

\section{FIG. 5 HERE}

3) Characterization of HDI Instability

A typical TD process depicted by AE RMS can be found in Fig. 6. The vibration amplitude shows three noticeable regions: flying, instability, and sliding. Before $114 \mathrm{~mW}$, the slider vibration is gentle, which is characterized by a stable flying. A bump around $115 \mathrm{~mW}-118 \mathrm{~mW}$ indicates a vigorous slider vibration, which is then suppressed by further increasing the TFC power. The bump in TD process is generally characterized as the flying instability region, in which the slider is bouncing from disk, or intermittently contacting with disk [25]. The suppressed vibration indicates that the slider is continuously sliding on the disk.

\section{FIG. 6 HERE}

The instability region in the TD process contains rich information about the slider-disk interaction. The propagation of this instability region indicates the situation of slider-disk interaction getting worse.

\section{RESULTS AND DISCUSSION}

\section{A. Slider Wear Progression}

The evolution of the head wear characterized by TDP loss is shown in Fig. 7. The whole wear path can be divided into three stages with respect to the wear rate. These three stages can reflect the evolution of slider and disk wear, as schematically presented in Fig. 8. In stage I, a fast wear rate is observed, indicating the wear mechanism being dominated by an abrasive wear [26]. The contact between disk asperities and slider protrusion contributes to this wear [27]. Because the contact intensity of the slider protrusion is much higher than the mating disk, the transition from roughness wear to geometry shape wear on the slider protrusion happened in a very short time, while the disk was still in a roughness wear stage. Stage II starts from 10 minutes, which is the changing point of the wear rate. The change in wear rate indicates that the wear mechanism transfers from abrasive wear to adhesive wear. This transition can be attributed to the burnishing of asperities. As the asperities are gradually burnished, the contact type between slider and disk also shifts from plasticdominant to elastic-dominant [27]. In stage III, the contact type becomes elastic-dominant since wear path become nearly flat. This means that the head-disk wear also came into the stage of adhesive wear. The characteristic of adhesive wear is that it does not remove material too much. Only when the interfacial adhesion stress is greater than the fracture strength of the surfaces, the fragment might be plucked off and attached to the harder surface. It is worthy to note that the adhesive wear occurred in our experiments did not produce 
much wear, which indicated that the interfacial adhesion stress was not too high compared to the fracture strength of the DLC film.

\section{FIG. 7 HERE}

\section{FIG. 8 HERE}

To verify the findings listed above, four sliders were inspected by SEM after 5-minute, 15-minute, 30-minute, and 100-minute overdrive tests, respectively. As seen from Fig. 9, the wear region started from writer shield, and then extended to reader shield. The contact areas in 5 minutes (Fig. 9 (a)) and 15 minutes (Fig. 9 (b)) are very rough, indicating that they were still in the stage of asperity burnishing. After 30-minute overdrive (Fig. 9 (c)) and 100-minute overdrive (Fig. 9 (d)), the contact areas are much smoother, indicating that the wear type has shifted from abrasive wear to adhesive wear. This agrees well with the three-stage wear progression illustrated in Fig. 7 and Fig. 8.

\section{FIG. 9 HERE}

The mating disk surface was also inspected by OSA after the overdrive test. Generally, QPhase channel of OSA is sensitive to the change in lubricant layer, while the PSc channel is sensitive to the change in carbon layer [10], [16][18]. Thus, we used QPhase channel to measure the lubricant depletion and modulation, and used PSc channel to detect the DLC wear. From Fig. 10, no detectable wear or scratch was observed after 15-minute and 100-minute overdrive tests, but depletion was observed on the lubricant layer. This indicates that when the slider was suffering from serious wear, the disk was still in the stage of asperity burnishing. It can be explained by the fact that disk has a much larger contact area than slider (the contact area of disk is the whole circular track while the contact area of slider is mainly fixed on the transducer area [10]).

\section{FIG. 10 HERE}

\section{B. The Propagation of HDI Instability}

The evolutions of AE and TA RMS plots are shown in Fig. 11 and Fig. 12 respectively. The flying instability region demonstrated a rapid increase in amplitude over wearing duration, indicating that a larger slider vibration became easier to be excited after a series of overdrive tests. In other words, the HDI stability was becoming worse along the wearing duration. The instability demonstrated by the "bouncing vibration" indicates that a nonlinear vibration, internal resonance [28], [29], was formed. The adhesion force between slider and lubricant is the main reason for the "bouncing vibration" [30], [31]. The propagation of slider wear promotes the contact area, and thus strengthens the adhesion force, which further leads to a larger contact force exerted by the disk on the slider and, hence, stronger "bouncing".

\section{FIG. 11 HERE}

\section{FIG. 12 HERE}

To investigate the causes of the instability propagation, the burst points, A, B, C, D, E, and F of AE RMS curves and A', $B^{\prime}, C^{\prime}, D^{\prime}, E^{\prime}$, and $F^{\prime}$ of TA RMS curves were explored in frequency domain. As shown in Fig. 13 (a) and Fig. 14 (a), the initial slider response is very gentle (notice that the magnitude is with logarithmic scale) and only $98 \mathrm{kHz}$ peak can be observed. After 2 minutes, AE spectrum (Fig. 13(b)) shows that $98 \mathrm{kHz}$ peak become dominant and its harmonic $(196 \mathrm{kHz})$ peak also appears. Compared to AE signal, TA signal (Fig. 14 (b)) shows much richer frequency content, that is, besides the harmonic peaks of $98 \mathrm{kHz}, 244 \mathrm{kHz}$ peak also appears. When the testing duration increases, more frequency components were observed in both $\mathrm{AE}$ and $\mathrm{TA}$ spectrums. After 10 minutes, AE signal (Fig. 13 (d)) shows that $244 \mathrm{kHz}$ peak becomes significant and $146 \mathrm{kHz}$ peak appears. The $244 \mathrm{kHz}$ component is indeed a sideband of $146 \mathrm{kHz}$. In other words, the $146 \mathrm{kHz}$ associated vibration is modulated by $98 \mathrm{kHz}$ associated vibration, which produces two sidebands of 146 kHz: $48 \mathrm{kHz}(146 \mathrm{kHz}-98 \mathrm{kHz})$ and $244 \mathrm{kHz}(146 \mathrm{kHz}+98$ $\mathrm{kHz}$ ). This is clearer in TA spectrums, as shown in Fig. 14 (d). After 15 minutes and 30 minutes, the frequency content shows no change, but the magnitudes of the frequency components increase.

\section{FIG. 13 HERE}

\section{FIG. 14 HERE}

To explore the frequency components observed in the spectrums, we conducted a modal analysis using the finite element method. The whole HGA was modeled by 3-D solid elements in ANSYS. The suspension and slider were modeled in details including the dimple, flexure, and solder balls, etc. The air bearing was modeled by four springs with different stiffnesses. As shown in Fig. 15, the strongest vibration response appears at about $95 \mathrm{kHz}$, which is close to the measured frequency $(98 \mathrm{kHz})$. By checking the mode shape of this frequency, it is a suspension-air-bearing coupled bending mode. Another strong response at $147 \mathrm{kHz}$ that is almost the same as the measured frequency $(146 \mathrm{kHz})$ is identified as the first pitch mode of the air bearing.

\section{FIG. 15 HERE}

From the analysis of the frequency spectrums presented above, we can find that the nonlinear effects occur in different levels with respect to the wear stages. The first appeared frequency component is only $98 \mathrm{kHz}$, which indicates that the contacting surfaces were still rough and the adhesion force between the mating surfaces were not too high. In other words, at this stage, no nonlinear effect occurred. As the wear extended, the contact areas became smoother and smoother, making the surface energy and adhesion force between the mating surfaces increase. The adhesion force drived the sliderldisk system into internal resonance. The evidence is that the harmonic peaks of $98 \mathrm{kHz}$ appearred in the spectrum. With the wear extending further, the modulation effect appearred. The modulation effect is caused by the interaction between the 
$98 \mathrm{kHz}$ mode and $\mathrm{P} 1$ mode $(146 \mathrm{kHz})$, i.e., the occurrence of the pair of $(146-98) \mathrm{kHz}$ and $(146+98) \mathrm{kHz}$. As suggested in the modal analysis, the mode shape of $98 \mathrm{kHz}$ is a suspension related bending mode, which is easily interacted with slider vertical motion, for example, the motion caused by air bearing pitch modes. Alternatively, a theory developed by Canchi et al. [32] can also be used to explain the modulation phenomena. According to the findings in [32], all the measured frequency components are the sub harmonics, super harmonics and fractional harmonics of the nonlinear system. Therefore, the $98 \mathrm{kHz}$ can be regarded as the dominant peak, and $146,48 \mathrm{kHz}$, and $244 \mathrm{kHz}$ are the $3 / 2,1 / 2$, and $5 / 2$ fractional harmonics, respectively. These peaks were thought to be the results of slider response to the periodic lubricant rippling and media waviness. Using these findings, we can extend our understanding of the relationship between the slider response and contact condition. In other words, when two rough surfaces are in contact (abrasive wear), the response might only consists of the low-frequency components, e.g., 98 $\mathrm{kHz}$, while when two smooth surfaces are in contact (adhesive wear), the response might consist of the nonlinear effects including the harmonics and the modulation effects, e.g., 196 $\mathrm{kHz}, 294 \mathrm{kHz}, 48 \mathrm{kHz}$ and $244 \mathrm{kHz}$.

\section{CONCLUSIONS}

This paper presented an in-situ assessment technique for HDI degradation using AE sensor and TA sensor. The wear mechanism shift and HDI instability propagation were experimentally demonstrated through monitoring the TD dynamics before and after each cycle of the overdrive test. The monitored TDP loss can quantify the head wear well and its evolution reveals that the wear mechanism is shifted from abrasive wear to adhesive wear with the increase of wear duration. The HDI instability characterized by the slider bouncing vibration reveals that the suspension-air-bearingcoupled mode dominated the instability evolution as its harmonics and modulating effect dominates the frequency spectrum. This instability is attributed to the adhesion force between slider and disk. The progression of slider/disk wear strengthens the adhesion force, and hence HDI instability. Therefore, besides improving the anti-wear performance of slider and disk, reducing the interfacial adhesion is also a critical task to mitigate the HDI degradation. In addition, the suspension and air bearing surface should be well designed to avoid the suspension-air bearing coupled bending mode.

\section{ACKNOWLEDGMENT}

The authors would like to thank the editor Prof. Bhatia and the anonymous reviewers for their valuable comments and suggestions that greatly improve this paper. The work described in this paper was supported by a grant from the Research Grants Council of the Hong Kong Special Administrative Region, China (CityU8/CRF/09) and Innovation and Technology Fund (GHX/003/12).

\section{REFERENCES}

[1] M. Hilbert and P. Lopez, "The world's technological capacity to store, communicate, and compute information," Science, vol. 332, no. 60, pp. $60-65,2011$.

[2] J. Juang, T. Nakamura, B. Knigge, Y. Luo, W. Hsiao, K. Kuroki, F. Huang, and P. Baumgart, "Numerical and experimental analyses of nanometer-scale flying height control of magnetic head with heating element," IEEE Trans. Magn., vol. 44, no. 11, pp. 3679-3682, Nov.2008.

[3] L. Su, Y. Hu, E. L. Lam, P. Li, R. W. Ng, D. Liang, O. Zheng, H Liu, Z. Deng, and J. Zhang, "Tribological and dynamic study of head disk interface at sub-1-nm clearance," IEEE Trans. Magn., vol. 47, no. 1, pp. 111-116, Jan. 2011.

[4] A. Khurshudov and P. Ivett, "Head-disk contact detection in the harddisk drives," Wear, vol. 255, pp. 1314-1322, 2003.

[5] B. D. Strom, S. C. Lee, G. W. Tyndall, and A. Khurshudov, "Hard disk drive reliability modeling and failure prediction," IEEE Trans. Magn., vol. 43, no. 9, pp. 3676-3684, Sept. 2007.

[6] R. R. Katta, A. A. Polycarpou, S. C. Lee, M. Suk, "Experimental and FEA scratch of magnetic storage thin-film disks to correlate magnetic signal degradation with permanent deformation," J. Tribol., vol. 132, pp. 021902-1-021902-11, Apr. 2010.

[7] T. Imaura and K. Yamamoto, "Degradation testing and lifetime prediction for GMR heads under mechanically and thermally accelerated conditions," IEEE Trans. Magn., vol. 41(10), pp. 3037-3039, Oct. 2005.

[8] J. G. Elerath and M. Pecht, "A highly accurate method for assessing reliability of redundant arrays of inexpensive disks (RAID)," IEEE Trans. Computers, vol. 58, no. 3, pp. 289-299, March 2009.

[9] A. Khurshudov and V. Raman "Roughness efects on head-disk interface durability and reliability," Tribol. Int., vol. 38, pp. 646-651, 2005.

[10] N. Li, L. Zheng, Y. Meng, and D.B Bogy, "Experimental study of headdisk interface flyability and durability at sub-1-nm clearance," IEEE Trans. Magn., vol. 45, no. 10, pp. 3624-3627, Oct. 2009.

[11] D. Song, R. Kvitek, and D. Schnur, "Inspection of pole tip diamondlike carbon wear due to heater-induced head-disc contact," J. Appl. Phys., vol. 99, 08N107-1-3, 2006.

[12] J. Juang, J. Forrest, and F. Huang, "Magnetic head protrusion profiles and wear pattern of thermal flying-height control sliders with different heater designs," IEEE Trans. Magn., vol. 47, no. 10, pp. 3437-3440, Oct. 2011.

[13] S. H. Kim, X. Guo, R. J. Waltman, H. Tu, T. Shatz, and D. J. Pocker, "Durability against contact wear of nonlubricated disks in the head-disk interface of disk drives," IEEE Trans. Magn., vol. 47, no. 1, pp. 239243, Jan. 2011

[14] B. Liu, M. S. Zhang, S. K. Yu, W. Hua, Y. S. Ma, W. D. Zhou, L. Gonzaga, and Y. J. Man, "Lube-surfing recording and its feasibility exploration," IEEE Trans. Magn., vol. 45, no. 2, pp. 899-904, Feb. 2009.

[15] Y. Chen, J. Zheng, and D. B. Bogy, "Light contact and surfing state dynamics of air bearing sliders in hard disk drives," Appl. Phys. Lett., vol. 100, 243104-1-4, 2012.

[16] R. Pit, B. Marchon, S. Meeks, and V. Velidandla, "Formation of lubricant "moguls" at the head/disk interface", Tribol. Lett., vol. 10, no. 3, pp. 133-142, 2001.

[17] S. Guddati, J. Zhang, S. K. Sinha, and S. M. Hsu, "Nanolubrication: Characterization of patterned lubricant films on magnetic hard disks," Tribol. Lett., vol. 21, no. 3, Mar. 2006.

[18] R-H Wang, S. W. Meeks, R. L. White, and W. E. Weresin, "The effect of hydrogen in carbon overcoats on the tribology of the head-disk interface," IEEE Trans. Magn., vol. 31, no. 6, pp. 2919-2921, Nov. 1995.

[19] Q. Zeng, C. Yang, S. Ka, and E. Cha, "An experimental and simulation study of touchdown dynamics," IEEE Trans. Magn., vol. 47, no. 10, pp. 3433-3436, Oct. 2011.

[20] Y. Shimizu, J. Xu, H. Kohira, K. Kuroki and K. Ono, "Experimental study on slider dynamics during touchdown by using thermal flyingheight control”, Microsyst Tech., vol. 17, pp. 897-902, 2011.

[21] S. V. Canchi and D. B. Bogy, "Slider dynamics in the lubricant-contact regime," IEEE Trans. Magn., vol. 46, no. 3, pp. 764-769, Mar. 2011.

[22] R. Sundaram, W. Yao, R. Ku, and D. Kuo, "Study of head/disc interface dynamics using a thermal asperity sensor," IEEE Trans. Magn., vol. 35, no. 5, pp. 2481-2483, Sept. 1999.

[23] Y. Shimizu, J. Xu, H. Kohira, M. Kuita, T. Shiramatsu, and M. Furukawa, "Nano-scale defect mapping on a magnetic disk surface using 
This article has been accepted for publication in a future issue of this journal, but has not been fully edited. Content may change prior to final publication. Citation information: DOI 10.1109/TMAG.2013.2286579, IEEE Transactions on Magnetics

$>$ FOR CONFERENCE-RELATED PAPERS, REPLACE THIS LINE WITH YOUR SESSION NUMBER, E.G., AB-02 (DOUBLE-CLICK HERE) <

a contact sensor," IEEE Trans. Magn., vol. 47, no. 10, pp. 3426-3432, Oct. 2011.

[24] X. Che, W. Huang, Z. Lin, A. Shteyn, "System and method for calibrating and controlling a fly-height actuator in a magnetic recording disk drive", U.S. Patent, 7180 692, Feb. 2007.

[25] J. Zheng, D.B. Bogy, "Numerical simulation of touchdown dynamics of thermal flying-height control sliders," IEEE Trans. Magn.,, vol.48, no.9, pp.2415-2420, Sept. 2012

[26] B. Bhushan, Principles and Application of Tribology, John Wiley \& Sons, Inc., New York, 1999.

[27] W. Peng, J. Kiely, and Y-T, Hsia, "Wear analysis of head-disk interface during contact," J. Tribol., vol. 127, pp. 171-179, Jan. 2005.

[28] S. K. Yu, B. Liu, K. K. Ng, W. Hua, W. D. Zhou, and K. S. Myo, "Nonlinear dynamics of thermal flying height control sliders at touchdown," IEEE Trans. Magn., vol. 47, no. 7, pp. 1798-1804, July 2011.

[29] K. Ono, M. Yamane, and H. Yamaura, "Experimental and analytical study of bouncing vibrations of a flying head slider in a near-contact regime," J. Tribol., vol. 127, no. 2, pp. 376-386, 2005.

[30] Y. Shimizu, K. Ono, N. Umehara, and J. Xu, "Experimental and numerical simulation study on low-surface energy slider with thermal flying-height control function," IEEE Trans. Magn., vol. 45, no. 10, pp. 3620-3623, 2009.

[31] J. Zheng and D. B. Bogy, "Investigation of flying-height stability of thermal fly-height control sliders in lubricant or solid contact with roughness," Tribol Lett, vol. 38, pp. 283-289, 2010.

[32] S. Canchi and D.B. Bogy, "Thermal fly-height control slider instability and dynamics at touchdown: explanations using nonlinear systems theory," J. Tribol., vol. 133, no. 2, pp. 021902:1-13, 2011.

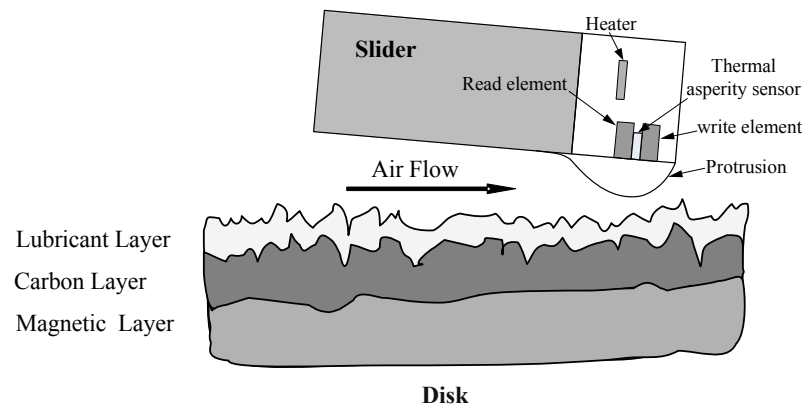

Fig. 1. Diagram of HDI in operational condition.

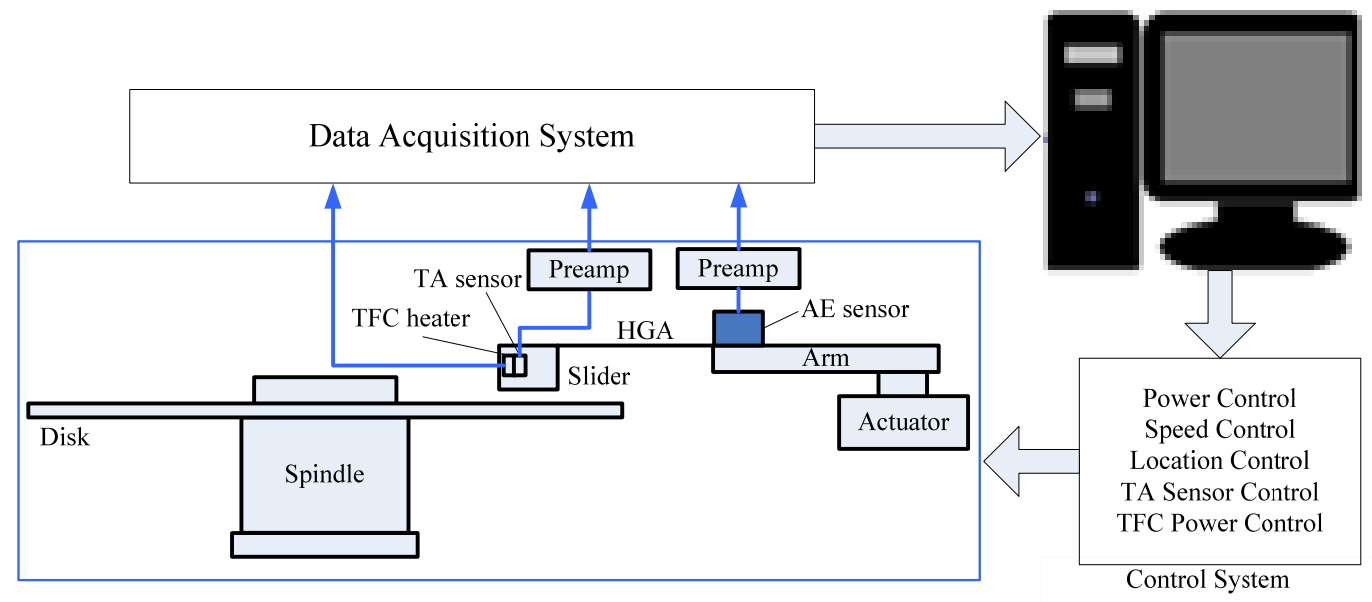

Fig. 2. Diagram of experimental setup. 
This article has been accepted for publication in a future issue of this journal, but has not been fully edited. Content may change prior to final publication. Citation information: DOI

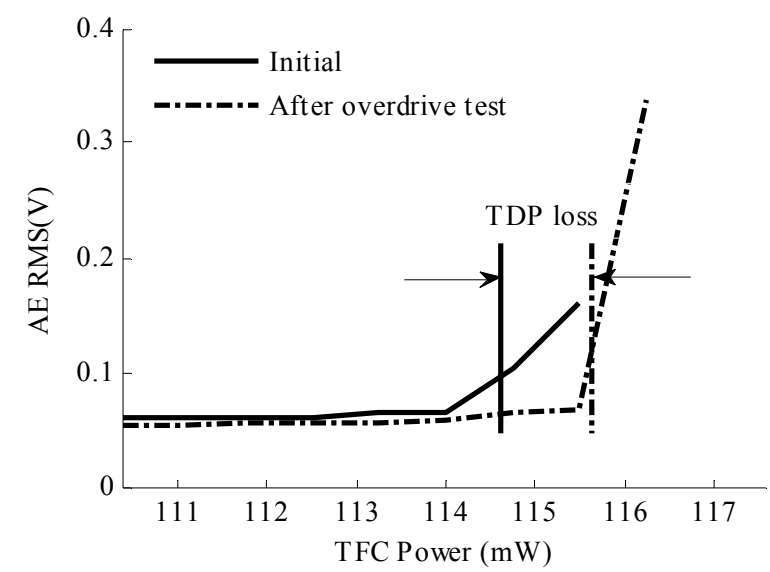

Fig. 3. AE RMS changed at initial status and after overdrive test. Power denotes the heater power. AE RMS was calculated for each power.
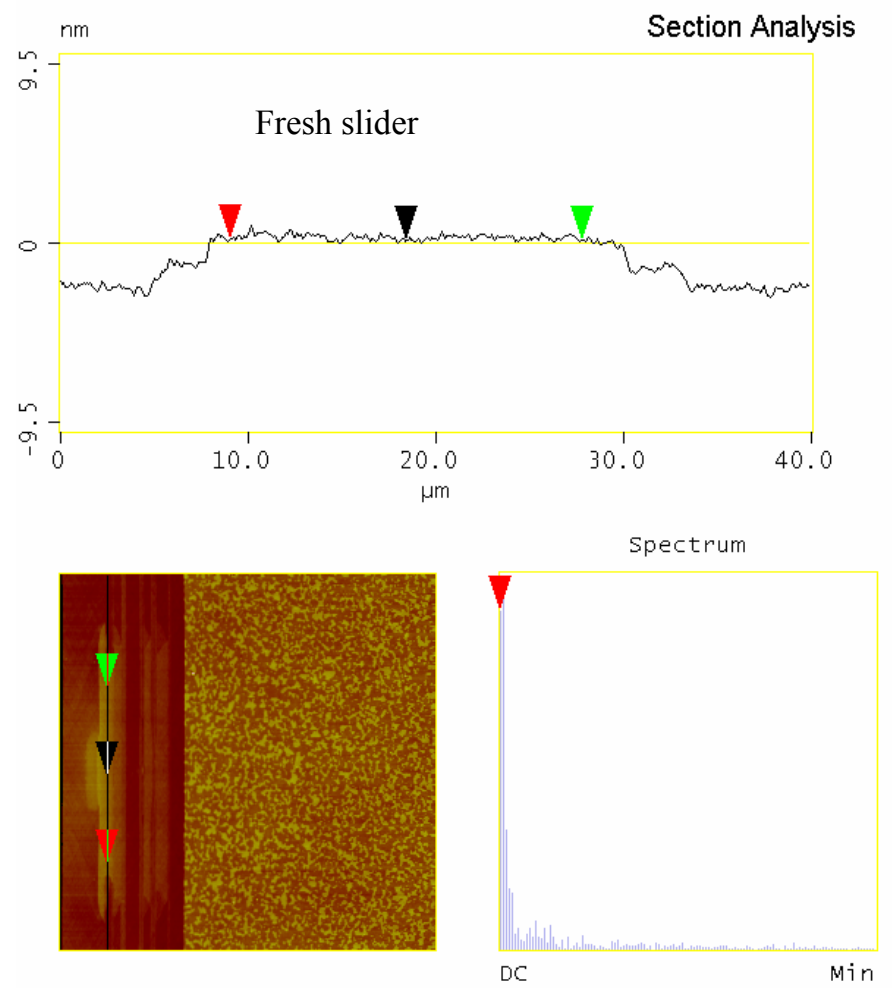

(a) $\stackrel{\circ}{0}^{\mathrm{n} m}$

Section Analysis

After overdrive test
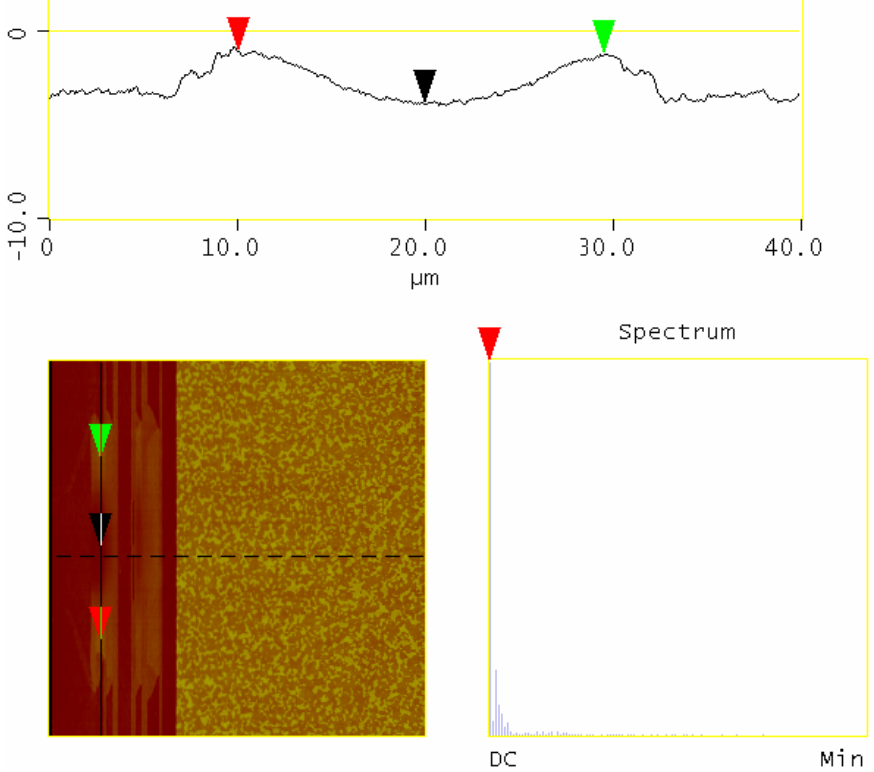

(b)

Fig. 4. Cross profile of writer shield measured by AFM. (a) The slider without wearing. (b) The slider after overdrive test.

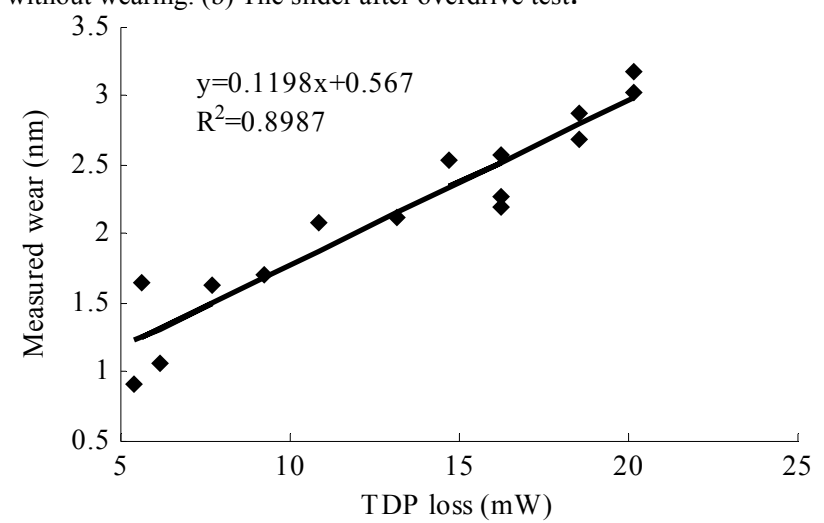

Fig. 5. Correlation between the TDP loss and real wear. Real wear depth was measured by AFM.

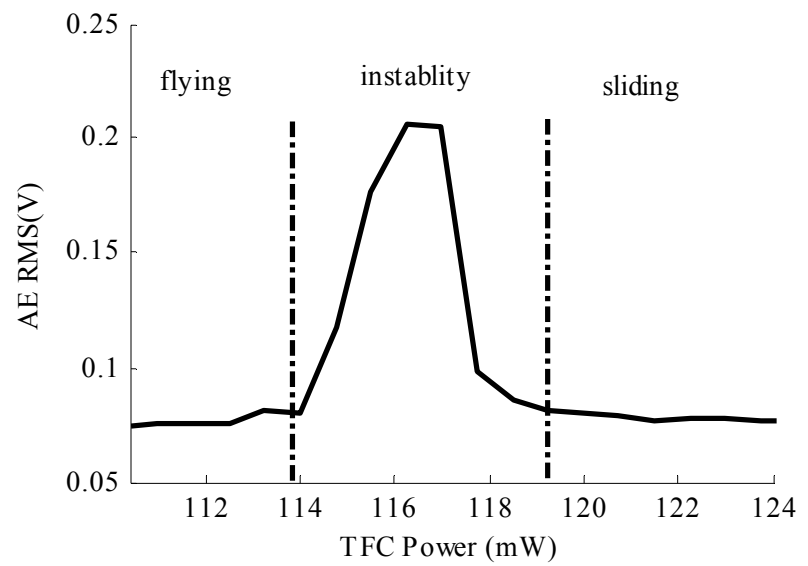

Fig. 6. A typical TD process includes three regions: flying region, instability region, and sliding region. 
This article has been accepted for publication in a future issue of this journal, but has not been fully edited. Content may change prior to final publication. Citation information: DOI 10.1109/TMAG.2013.2286579, IEEE Transactions on Magnetics

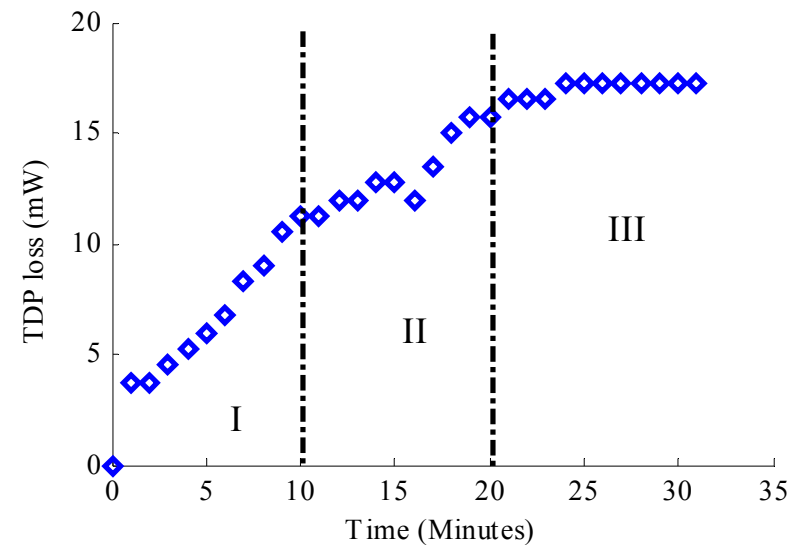

Fig. 7. Monitored head wear characterized by TDP loss

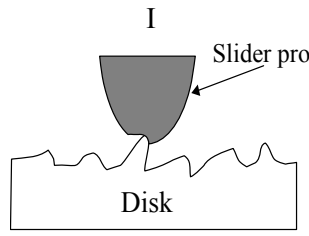

(a)
II

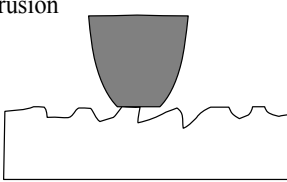

(b)
III

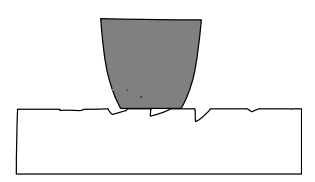

(c)
Fig. 8. Three stages of wearing. (a) Stage I, abrasive wear; (b) Stage II, abrasive and adhesive wear; and (c) Stage III, adhesive wear.

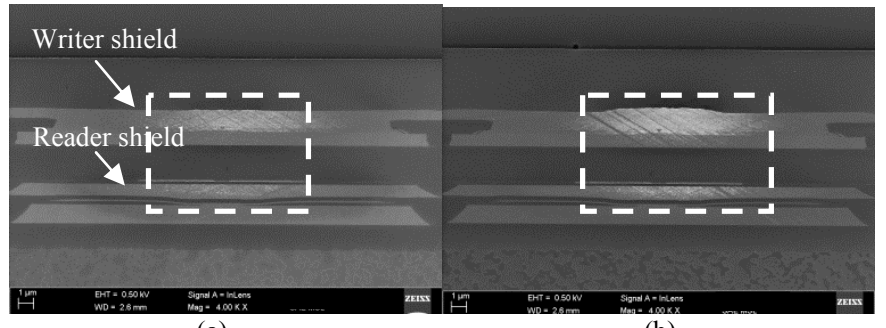

(a)

(b)

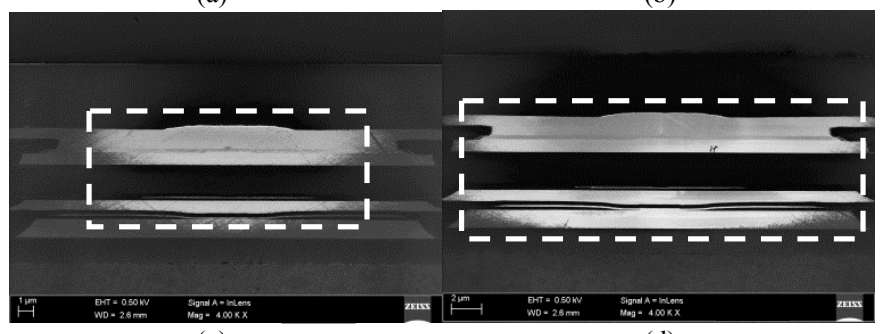

(c)

(d)

Fig. 9. The SEM results of different stages. (a) 5 minutes; (b) 15 minutes (c) 30 minutes; and (d) 100 minutes. The boxes in dash show the wear areas of the sliders.

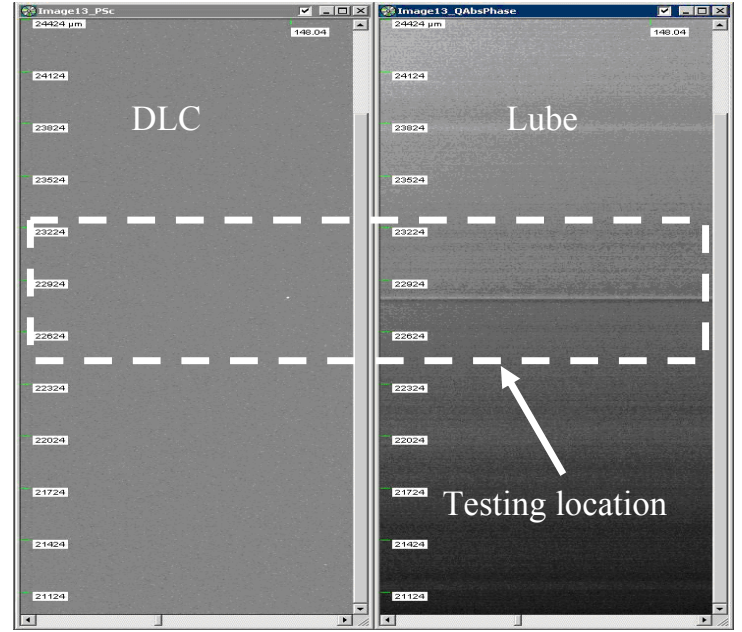

(a)

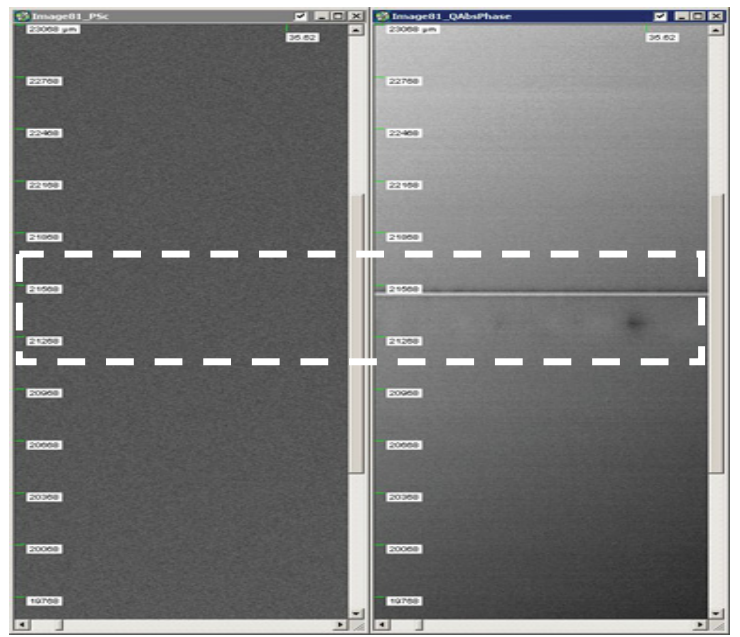

(b)

Fig. 10. OSA inspection for disk. No detectable wear was found in DLC layer. Lube depletion was observed in lube layer. (a) After 15 minutes overdrive test; and (b) After 100 minutes overdrive test

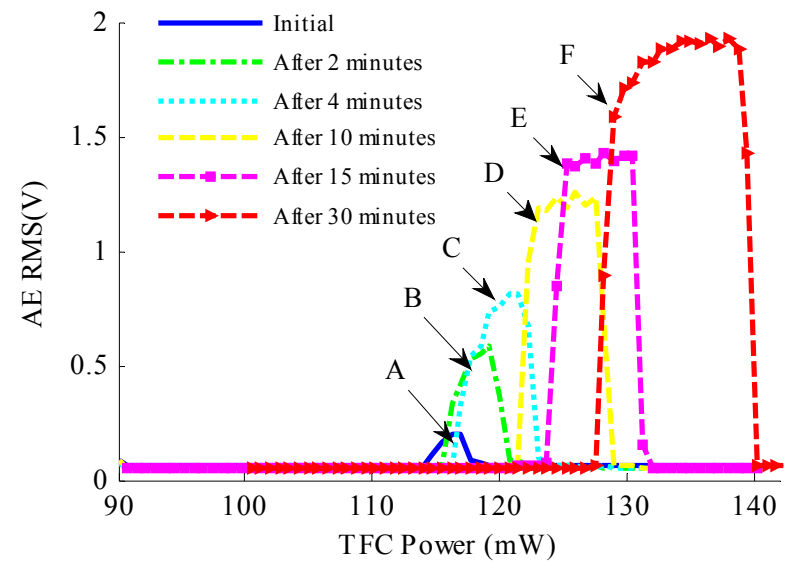

Fig. 11. AE RMS variation with wearing time. A, B, C, D, E and F are the burst points. 


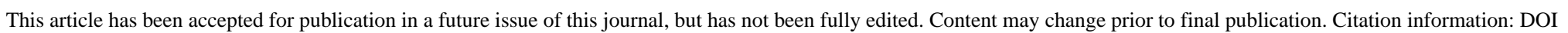

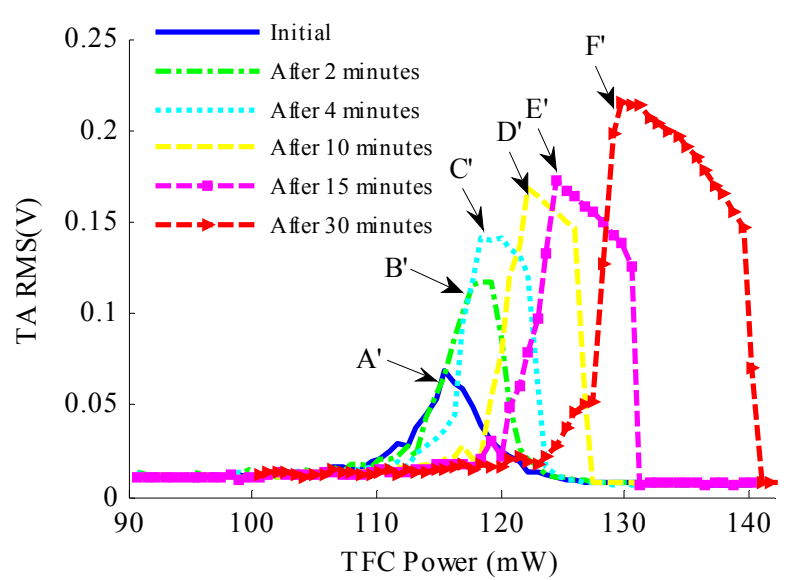

Fig. 12. TA RMS variation with wearing time. $\mathrm{A}^{\prime}, \mathrm{B}^{\prime}, \mathrm{C}^{\prime}, \mathrm{D}^{\prime}, \mathrm{E}^{\prime}$, and $\mathrm{F}^{\prime}$ are the burst points.

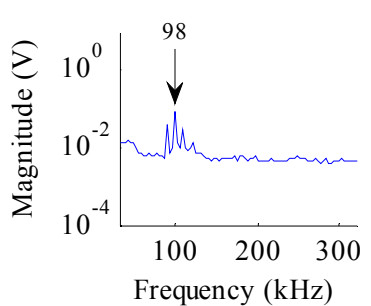

(a)

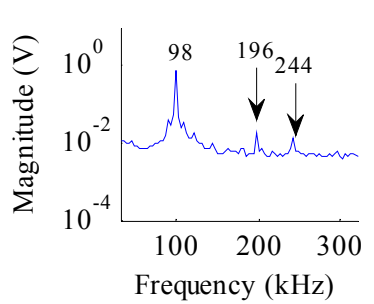

(c)

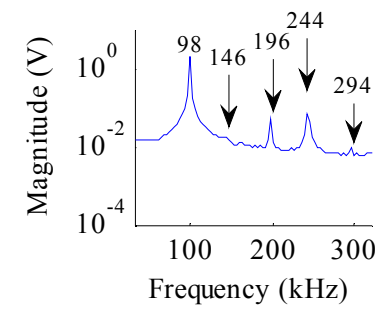

(e)

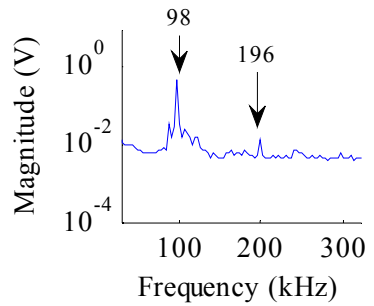

(b)

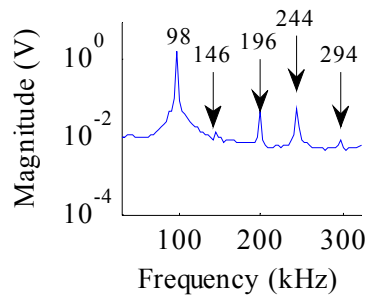

(d)

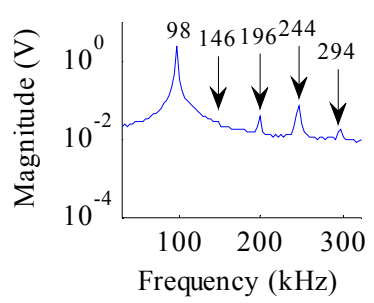

(f)
Fig. 13. Spectrum analysis for AE signals. (a) Spectrum of point A; (b) Spectrum of point B; (c) Spectrum of point C; (d) Spectrum of point D; (e) Spectrum of point E, and (f) Spectrum of point F.

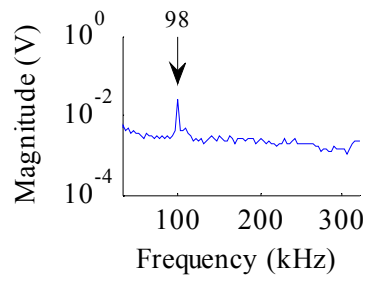

(a)

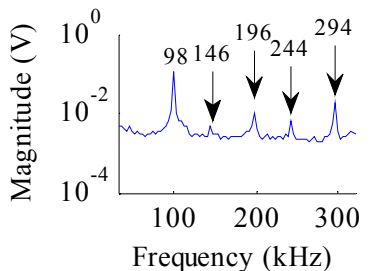

(c)

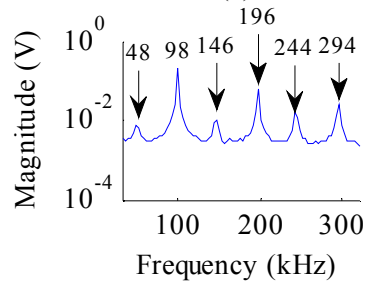

(e)

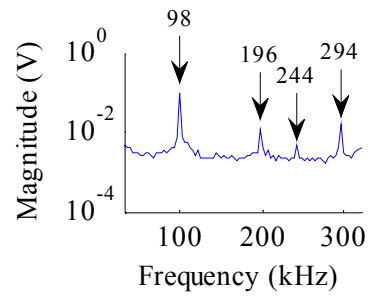

(b)

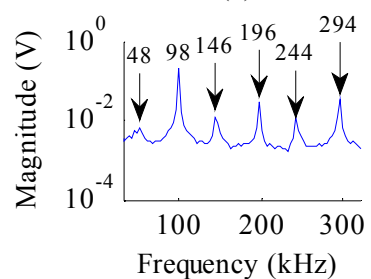

(d)

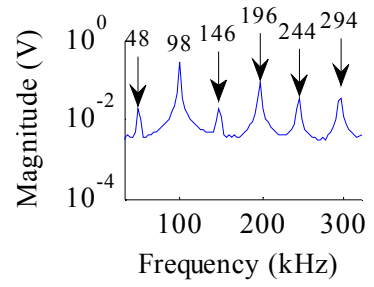

(f)
Fig. 14. Spectrum analysis for TA signals. (a) Spectrum of point A'; (b) Spectrum of point B'; (c) Spectrum of point C'; (d) Spectrum of point D'; (e) Spectrum of point E'; and (f) Spectrum of point F'.

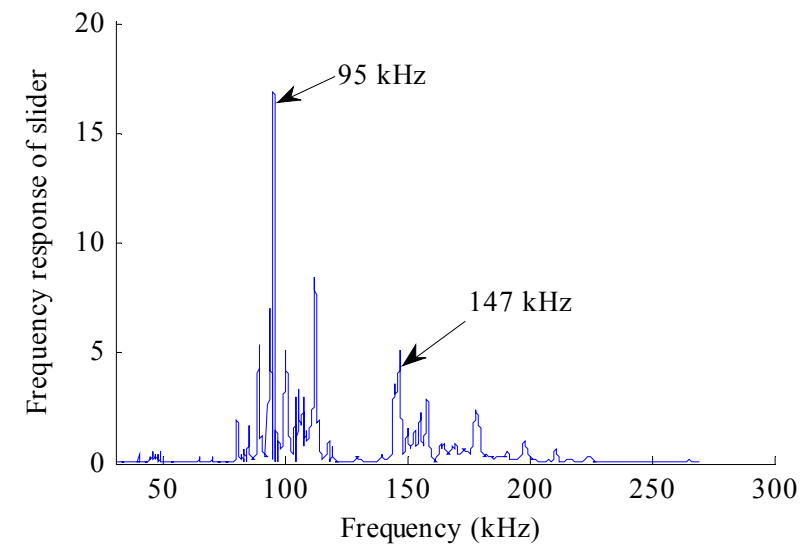

Fig. 15. Simulation of slider response during touch down 